\title{
Erratum to: Thrombectomy for ischemic stroke: meta-analyses of recurrent strokes, vasospasms, and subarachnoid hemorrhages
}

\author{
Robert Emprechtinger $^{1}$ (D) Brigitte Piso $^{1} \cdot{\text { Peter A. } \text { Ringleb }^{2}}^{2}$
}

Published online: 9 January 2017

(c) Springer-Verlag Berlin Heidelberg 2017

\section{Erratum to: J Neurol \\ DOI 10.1007/s00415-016-8205-1}

The authors would like to correct the errors in the publication of the original article. The corrected details are given below for your reading.

In the results section, the second paragraph should read as:

MR CLEAN, ESCAPE, REVASCAT, and SWIFT PRIME reported the number of subarachnoid hemorrhages $[1,2,4,5]$. In all trials, these were more frequent in the intervention groups than in the control groups. Endovascular therapy led to a statistically significant higher rate of subarachnoid hemorrhage, regardless of whether the fixed effects model (RR 3.27, 95\% CI 1.08-9.91, $p=0.036$ ), or the random effects model (RR 3.19, 95\% CI 1.04-9.76, $p=0.042$ ) was considered (Fig. 2).

The online version of the original article can be found under doi:10.1007/s00415-016-8205-1.

Robert Emprechtinger

robert.emprechtinger@hta.lbg.ac.at

Brigitte Piso

brigitte.piso@hta.lbg.ac.at

Peter A. Ringleb

peter.ringleb@med.uni-heidelberg.de

1 Ludwig Boltzmann Institute for Health Technology Assessment, Garnisongasse 7, 1090 Vienna, Austria

2 Department of Neurology, University Hospital Heidelberg, Im Neuenheimer Feld 400, 69120 Heidelberg, Germany 